\title{
Comparison of optical coherence tomography angiography features in type 1 versus type 2 choroidal neovascular membranes secondary to age-related macular degeneration
}

\author{
Yasin Sakir Goker ${ }^{1}$ and Gokhan Demir ${ }^{2}$ \\ ${ }^{1}$ Ophthalmology Department, Ulucanlar Eye Training and Research Hospital, Ankara, Turkey \\ ${ }^{2}$ Fatih Sultan Mehmet Training and Research Hospital, Istanbul, Turkey
}

\begin{abstract}
Background: Optical coherence tomography angiography (OCTA) is an advanced imaging modality that provides high resolution images at the level of different retinal layers. This study aime to evaluate choroidal neovascular membranes (CNVMs) secondary to age-related macular degeneration (AMD) quantitatively and qualitatively, according to their classification, morphological features, and flow areas, using OCTA.

Methods: In this descriptive, comparative, cross-sectional study, CNVMs were divided into 2 groups according to their classification as type 1 or type 2 neovascularization. Mixed CNVMs were excluded from the study. The size $\left(\mathrm{mm}^{2}\right)$ and the flow area $\left(\mathrm{mm}^{2}\right)$ of the CNVMs were calculated via OCTA and the presence of the perivascular halo and loop anastomoses were analyzed. The morphological appearance of the CNVMs were classified as: medusa, sea-fan, lacy-wheel, glomerular, dead tree, and mature vascular networks.

Results: Of the 85 eyes assessed for eligibility, 45 eyes of 34 individuals with CNVM were enrolled in this retrospective study. Twenty-eight eyes had type 1 and 17 eyes had type 2 CNVMs. The mean size and flow area were greater in type 1 than in type $2 \mathrm{CNVMs}$ (mean \pm standard deviation [SD], $6.69 \pm 4.54$ and $3.61 \pm 3.56$ $\mathrm{mm}^{2}$ versus $3.04 \pm 1.98$ and $1.77 \pm 1.62 \mathrm{~mm}^{2} ; P=0.044$ and 0.046 , respectively). Among the 22 eyes with type 1 CNVMs and the 9 eyes with type 2 CNVMs, 31 eyes had exudative membranes. Among the eyes with exudative CNVMs, 22 eyes had a perivascular halo and 22 eyes had loop anastomoses; this was significantly more than in the non-exudative eyes ( $P=0.042$ and 0.041 , respectively). The lacy-wheel (38.7\%) and dead tree $(71.4 \%)$ patterns were the most frequent morphological appearance of the CNVMs in the exudative and non-exudative membranes, respectively.

Conclusions: OCTA provides objective documantation about CNVMs. A perivascular dark halo around CNVMs could be a criterion to define exudative membranes activity.

\section{KEYWORDS}

choroidal neovascular membrane, CNVM, flow area, optical coherence tomography angiography, loop anastomoses, perivascular dark halo
\end{abstract}

Correspondence: Gokhan Demir, M.D. Fatih Sultan Mehmet Training and Research Hospital, Ankara, 06240, Turkey. Email: dr.gkhndmr@gmail.com. ORCID iD: https://orcid.org/0000-0002-3734-1496

How to cite this article: Goker YS, Demir G. Comparison of optical coherence tomography angiography features in type 1 versus type 2 choroidal neovascular membranes secondary to age-related macular degeneration. Med Hypothesis Discov Innov Ophthalmol. 2021 Summer; 10(2): 67-73. https://doi. org/10.51329/mehdiophthal1423

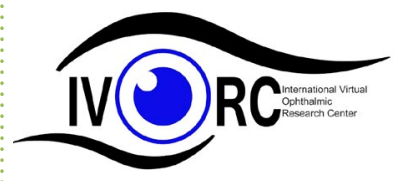

Copyright (C) Author(s). This is an open-access article distributed under the terms of the Creative Commons Attribution-NonCommercial 4.0 International License (http://creativecommons.org/licenses/by-nc/4.0/) which permits copy and redistribute the material just in noncommercial usages, provided the original work is properly cited. (c) (i) (s) 


\section{INTRODUCTION}

Choroidal neovascular membranes (CNVMs) represent a serious pathology that cause blindness, mostly in older patients. Its main etiopathogenesis is angiogenesis [1]. The most common cause of CNVM in developed countries is age-related macular degeneration (AMD) [2]. There are 3 types of CNVMs according to the location of the neovascular complex. Type 1 is the most frequent type, where the neovascular complex is below the retinal pigment epithelium (RPE). In type $2 \mathrm{CNVM}$, the lesion is above the RPE. Both these types originate from the choroid [3]. In type 3 lesions, the neovascularization originates from the deep capillary plexus; and is classified clinically as retinal angiomatous proliferation or type 3 neovascularization, rather than as a CNVM $[4,5]$.

Optical coherence tomography angiography (OCTA) is an advanced imaging modality that provides high resolution images at the level of different retinal layers [6]. This segmental data allows easy classification of CNVMs. Moreover, OCTA provides qualitative data, including morphological pattern, presence of a perivascular halo, and loop anastomoses, as well as quantitative data, including flow area and the greatest linear dimension, on CNVMs [7-10].

In the present study we aimed to evaluate CNVMs secondary to AMD quantitatively and qualitatively, according to their classification, morphological features, and flow areas, using OCTA.

\section{METHODS}

Consecutive cases with CNVMs secondary to AMD, who were followed from December 2017 through September 2019 at the Ulucanlar Eye Research and Education Hospital, Ankara, Turkey, were retrospectively evaluated. In this descriptive, comparative, cross-sectional study, all procedures were performed in accordance with the ethical standards of the institutional and/or national research committee and with the tenets of the 1964 Declaration of Helsinki and its later amendments, or comparable ethical standards. The study protocol was approved at the department level, and informed consent was obtained from all individual participants included in the study.

All study participants underwent complete ophthalmic examinations, including measurement of best corrected visual acuity according to a Snellen chart, evaluation of the anterior segment, intraocular pressure measurement with a Goldman applanation tonometer (Model AT 900 C/M; Haag-Streit, Köniz, Switzerland), and dilated fundus assessment with a $+90 \mathrm{D}$ lens (Volk Optical Inc, Mentor, OH, USA). Additionaly, the following imaging modalities were performed on the same day: spectral domain optical coherence tomography (SD-OCT) (Spectralis, Heidelberg Engineering Inc., Heidelberg, Germany), fundus fluorescein angiography (FFA) (Carl Zeiss Meditec, Dublin, CA, USA), and OCTA (Opto-Vue, Inc., Fremont, CA, USA).

Treatment-naive patients with CNVMs secondary to AMD were included in this study. These patients were divided into 2 groups according to their CNVM classification: patients with type 1 CNVM, and patients with type 2 CNVM. The classification was performed according to the automated outer retinal segmentation and choriocapillaris segmentation via OCTA. The study was designed in 2 parts: the first part was a quantitative evaluation, and the second part was a qualitative evaluation. For quantitative evaluation, we measured the size $\left(\mathrm{mm}^{2}\right)$ and the flow area $\left(\mathrm{mm}^{2}\right)$ of type 1 and type $2 \mathrm{CNVMs}$, while for a qualitative evaluation, we assessed the morphological characteristics among exudative and non-exudative groups. Retinal edema, hemorrhage, exudates and/or RPE detachments on fundus examination, and intraretinal, subretinal, and/or sub-RPE fluid accumulation on SD-OCT were considered as signs of exudative membranes.

Patients with mixed CNVMs were excluded from the study. Other exclusion criteria were: a history of previous anti-vascular endothelial growth factor (anti-VEGF) therapy, patients with glaucomatous findings (including IOP $>20$ millimeter of mercury, optic disc cupping, and optic disc haemorrhage), the presence of high myopia (>6 diopters), myopic CNVMs, history of previous photodynamic therapy, angioid streaks, macular telangiectasia, central serous chorioretinopathy, type $3 \mathrm{CNV}$ lesions, mixed choroidal neovascular membrane, pachychoroid neovasculopathy, polypoidal choroidal vasculopathy, and idiopathic CNV.

We performed OCT-A assessments as follows. Speckle noise removal and CNVM blood flow delineation were automatically performed while acquiring the OCTA image. CNVMs were evaluated quantitatively via the flow assessment tool of the OCTA device. The CNVM margins were selected manually by the observer and the device automatically calculated the size $\left(\mathrm{mm}^{2}\right)$ and the flow area $\left(\mathrm{mm}^{2}\right)$ of the CNVM (Figure 1). The morphological appearance of the CNVMs were classified as: medusa, sea-fan, lacy-wheel, glomerular, dead tree, and mature vascular networks. Medusa and sea-fan appearances were identified when the vessels branched in all directions from the center of the membrane (medusa) or from 1 side of the membrane (sea-fan) [11]. Lacy-wheel neovascularizations were described as well-circumscribed circular networks [12]. Dead tree or pruned vascular tree and mature vascular network patterns were identified as long, dilated, looped vessels without branching small capillary networks [13]. 
Glomerular morphology was described as similar to a glomerula in a nephron, as suggested by the name. Moreover the presence of a perivascular halo (Figure 2) and loop anastomoses were evaluated [14]. Patients $(n=9)$ with media opacity leading to low signal strength index $(<7)$ that obscured the view of the CNVM via OCTA were excluded from the study.

Statistical analysis was performed using SPSS software for Windows, version 18.0 (SPSS Inc., Chicago, IL, USA). The normality of the data distribution was verified with the Kolmogorov-Smirnov test $(P>0.05)$. Categorical variables are shown as numbers; numerical variables are shown as mean and standard deviation (SD). Size and flow area measurements of CNVMs were compared between groups using the Mann-Whitney test. Moreover, perivascular halo and loop anastomoses were compared between exudative and non-exudative CNVMs with the Mann-Whitney test. A $P$ value $<0.05$ was considered statistically significant.

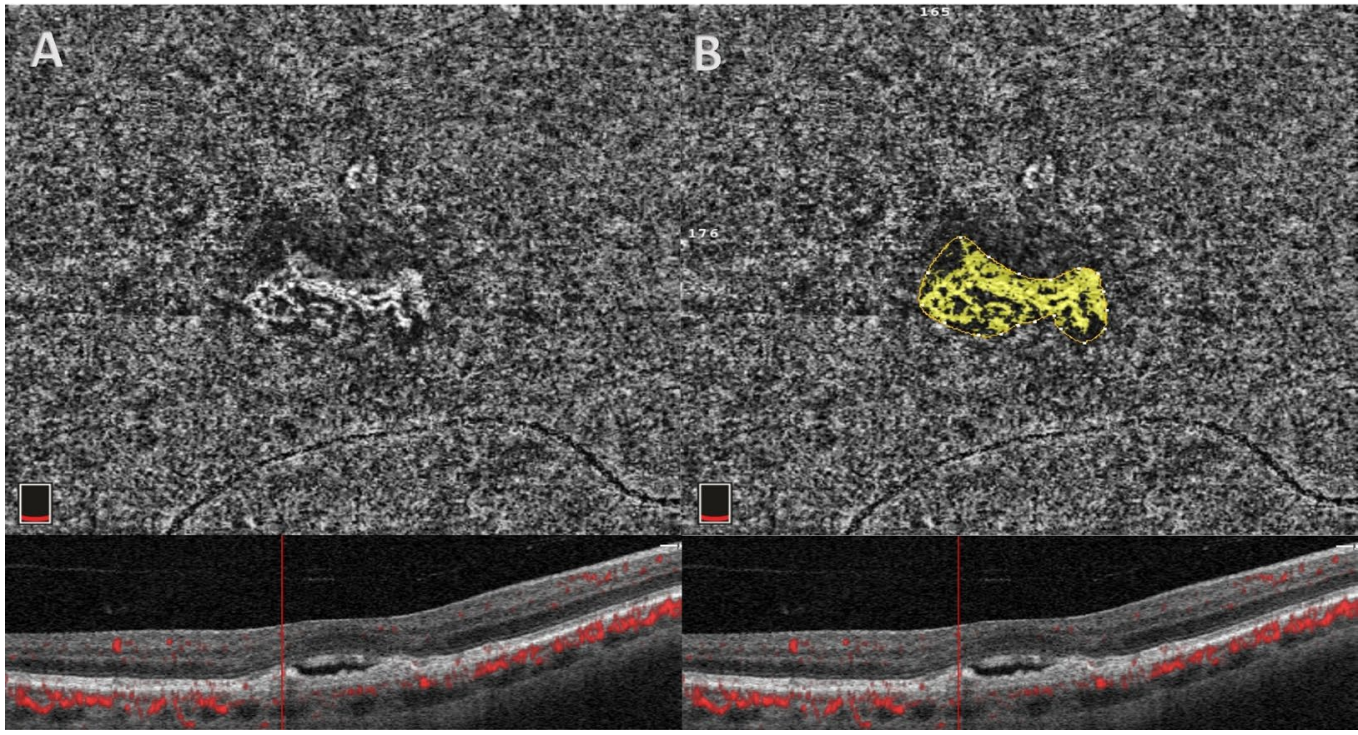

Figure 1. (A) A choroidal neovascular membrane (CNVM) was detected in the choriocapillaris segmentation of optical coherence tomography angiography. (B) The margins of the CNVM were selected manually by the observer and the device automatically calculated the size $\left(\mathrm{mm}^{2}\right)$ and the flow area $\left(\mathrm{mm}^{2}\right)$ of the membrane; which in this example was $1.215 \mathrm{~mm}^{2}$ and $0.708 \mathrm{~mm}^{2}$, respectively.

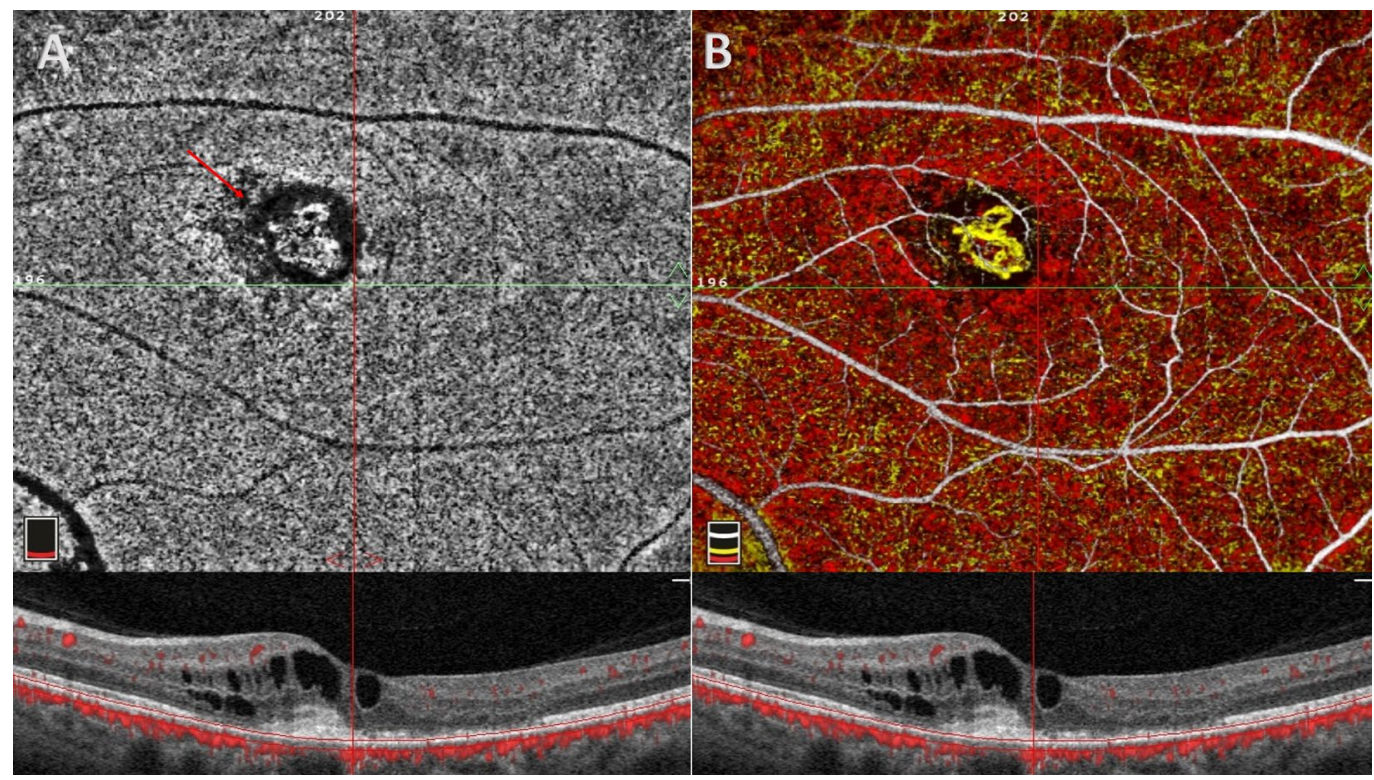

Figure 2. (A) The presence of a perivascular halo was evaluated in the choriocapillaris segmentation of optical coherence tomography angiography. (B) The color overlay image of the same patient. 


\section{RESULTS}

Of the 85 eyes assessed for eligibility, 45 eyes of 34 individuals (15 male, 19 female) with CNVM secondary to AMD were enrolled in the study. Figure 3 shows the flow diagram of subject eligibility. Table 1 summarizes the baseline demographic features in both groups. The mean \pm SD of age was $71.40 \pm 8.61$ years (range: $51-84$ years) for study participants overall. Twenty-eight eyes had type 1 CNVM (group 1) and 17 eyes had type 2 CNVM (group 2), with comparable baseline charateristics (Table 1).

In group 1, 22 eyes were in the active phase, 4 eyes were in the remission phase, and 2 eyes were in the quiescent phase. In group 2, 9 eyes were in the active phase, 6 eyes were in the remission phase, and 2 eyes were in the quiescent phase. Exudative membranes were present in 22 eyes with type 1 CNVMs and 9 eyes with type 2 CNVMs. Table 2 shows the qualitative and quantitative characteristics of study participants. Among the exudative CNVMs, 22 eyes had a perivascular halo, and 22 eyes had loop anastomoses; these numbers were significantly greater in exudative than in non-exudative CNVMs $(P=0.042$, and 0.041 respectively; Table 2). Moreover, all exudative type 2 CNVMs (100\%, 9 eyes) had perivascular halo.

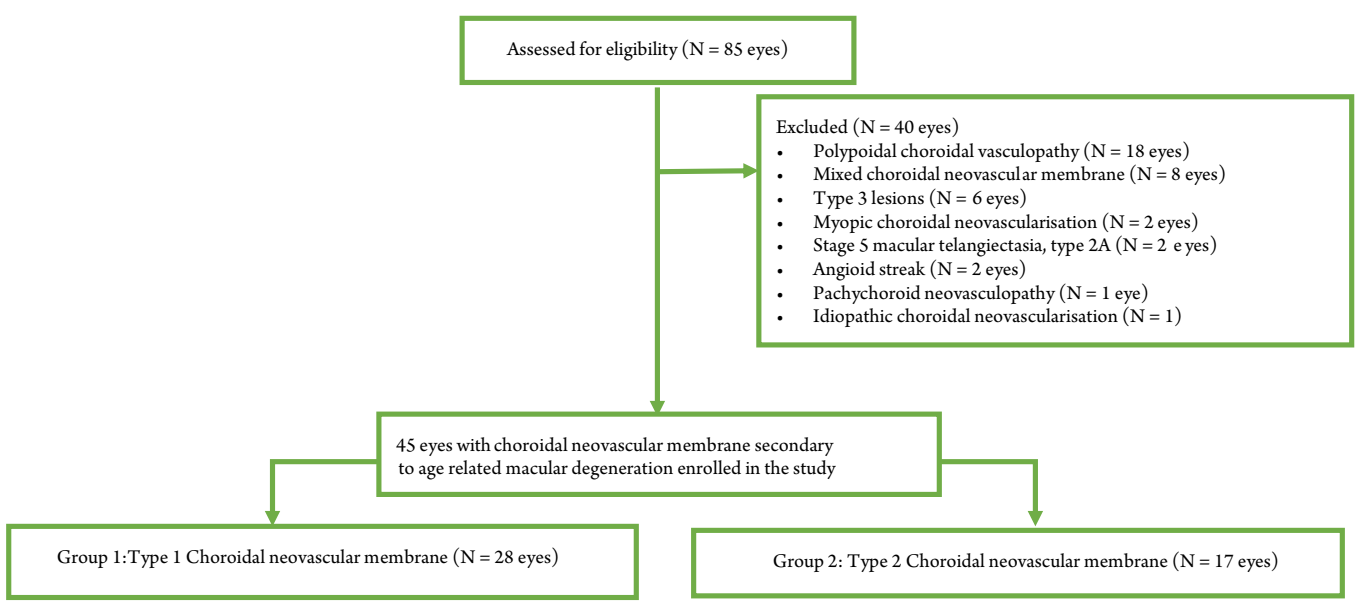

Figure 3. Flow diagram of patient eligibility.

Table 1. Baseline demographic characteristic features in both groups

\begin{tabular}{|c|c|c|c|}
\hline Variable & $\begin{array}{l}\text { Eyes with type } 1 \mathrm{CNVM} \\
(\mathrm{n}=28)\end{array}$ & $\begin{array}{l}\text { Eyes with type } 2 \text { CNVM } \\
(n=17)\end{array}$ & $P$-value \\
\hline Age $(y)$, Mean \pm SD & $70.2 \pm 5.1$ & $72.3 \pm 6.5$ & $0.35^{*}$ \\
\hline $\operatorname{Sex}(M / F), n$ & $8 / 10$ & $7 / 9$ & $0.39^{+}$ \\
\hline BCVA (decimal), Mean \pm SD & $0.3 \pm 0.21$ & $0.35 \pm 0.19$ & $0.45^{\ddagger}$ \\
\hline IOP $(\mathrm{mmHg})$, Mean \pm SD & $17.5 \pm 4.6$ & $16.4 \pm 4.2$ & $0.51^{\ddagger}$ \\
\hline
\end{tabular}

Abbreviatons: n, number; y, years; SD, standard deviation; M: Male, F: Female, CNVM, Choroidal neovascular membrane; BCVA, best corrected visual acuity; IOP, intraocular pressure; mmHg, millimeter of mercury; ${ }^{*}$ Independent samples t-test; ${ }^{\dagger} \mathrm{Chi}^{-s q u a r e d}$ test; ${ }^{\ddagger} \mathrm{Mann}{ }^{-}$ Whitney $\mathrm{U}$ test.

Table 2. The qualitative and quantitative characteristics of study participants

\begin{tabular}{|c|c|c|c|}
\hline \multicolumn{4}{|c|}{ Quantitative Features of Choroidal Neovascular membranes } \\
\hline Variable & $\begin{array}{l}\text { Type } 1 \text { CNVM } \\
(\mathrm{n}=28 \text { eyes })\end{array}$ & $\begin{array}{l}\text { Type } 2 \text { CNVM } \\
(\mathrm{n}=17 \text { eyes })\end{array}$ & $P$-Value \\
\hline Size $\left(\mathbf{m m}^{2}\right)$ & $6.69 \pm 4.54$ & $3.04 \pm 1.98$ & 0.044* \\
\hline Flow area $\left(\mathbf{m m}^{2}\right)$ & $3.61 \pm 3.56$ & $1.77 \pm 1.62$ & $0.046^{*}$ \\
\hline \multicolumn{4}{|c|}{ Qualitative Features of Choroidal Neovascular Membranes } \\
\hline Variable & $\begin{array}{l}\text { Exudative membranes } \\
\quad(n=31 \text { eyes })\end{array}$ & $\begin{array}{l}\text { Non-exudative membranes } \\
(\mathrm{n}=14 \text { eyes })\end{array}$ & $P$-Value \\
\hline Perivascular Halo (+/-) & $22 / 9$ & $3 / 11$ & $0.042^{*}$ \\
\hline Loop anastomoses $(+/-)$ & $22 / 9$ & $2 / 12$ & 0.041* \\
\hline
\end{tabular}

Abbreviations: CNVM, the choroidal neovascular membranes; $\mathbf{n}$, number; $\mathbf{~ m m}^{2}$, square millimeter; +, presence of perivascular halo or loop anastomoses; -, absence of perivascular halo or loop anastomoses; ${ }^{*}$ Mann Whitney test. $P$-value $<0.05$ is shown in bold. 
The mean size and flow area were greater in type 1 than in type $2 \mathrm{CNVMs}$ (mean $\pm \mathrm{SD}, 6.69 \pm 4.54 \mathrm{~mm}^{2}$ and $3.61 \pm 3.56 \mathrm{~mm}^{2}$ versus $3.04 \pm 1.98 \mathrm{~mm}^{2}$ and $1.77 \pm 1.62 \mathrm{~mm}^{2} ; P=0.044$ and 0.046 , respectively) (Table 2).

The morphological appearance of the CNVMs were: 12 eyes with lacy-wheel $(38.7 \%)$, 5 eyes with mature vascular network (16.1\%), 5 eyes with medusa (16.1\%), 4 eyes with glomerular (12.9\%), 2 eyes with seafan (6.4\%), and 3 eyes with an unidentifible pattern (9.6\%) among the exudative group. In the non-exudative group, 10 eyes had a dead tree (71.4\%), 1 eye had a glomerular (7.1\%), and 3 eyes had an unidentifiable pattern $(21.4 \%)$ of CNVM.

\section{DISCUSSION}

Our study demonstrated that the flow area and size were significantly greater in type 2 than in type 1 CNVMs and significantly more eyes with exudative CNVMs than non-exudative CNVs had a perivascular dark halo and loop anastomoses. The most frequent morphological appearance of exudative and non-exudative CNVMs were lacy-wheel $(38.7 \%)$ and dead tree $(71.4 \%)$, respectively.

Jung et al. and Cohen et al. evaluated the incidence of CNVMs and they reported that the most frequent type was type 1 CNVMs, or occult membranes, based on FFA classification [3, 15]. Type 1 and type 2 lesions had different staining patterns, according the location of the membrane. SD-OCT also provides information about the location and the activation of quiescent neovascularizations [16]. In the present study, we evaluated CNVMs with multimodal imaging modalities, including FFA, SD-OCT, and OCTA, and most (28 eyes, 62\%) had type 1 lesions, which was as consistent with the current literature.

Zhao et al. compared the quantitative features of type 1 and type 2 CNVMs via OCTA and reported that type 2 lesions had a smaller flow area and smaller greatest linear dimension [17]. We obtained similar results. The mean size and flow area of the type 1 CNVMs were significantly greater than those of type 2 CNVMs. Type 1 membranes are located below the RPE and they are mostly asymptomatic until they reach the neurosensory retina, which indicates a longer period of pathology. We hypothize that this asymptomatic period allows the membrane to become larger. However, in type 2 membranes, the pattern of spread is different. When the RPE is ruptured, the patient exhibits visual deterioration at an early stage. In type 2 CNVMs, the duration of the pathology and the length of time before it becomes symptomatic is shorter than in type 1 lesions [17].

Kuehlewein and colleagues used OCTA to analyze the morphology of type 1 CNVMs secondary to AMD and reported 2 morphological patterns: Medusa and Seafan [11]. El Ameen et al. evaluated type 2 CNVMs and reported that glomerulus-shaped and medusa-shaped membranes appear to be associated with type 2 CNVMs [14]. In contrast, of the 5 eyes with glomerulus- and the 5 eyes with medusa-shaped patterns in the present study, 4 and 3, respectively, were type 1 CNVMs.

Miere et al. also used OCTA to assess the morphological features of subretinal fibrosis secondary to AMD and described 3 vascular patterns: pruned vascular tree, tangled network, and vascular loop [8]. Twentysix of 49 eyes (53.1\%) with subretinal fibrosis had pruned vascular tree morphology and these eyes did not show any exudative symptoms via SD-OCT in the last 6 months [8]. Coscas and colleagues estimated 90.5\% correspondance between dead tree membrane appearance on OCTA and not requiring treatement group on conventional multimodal imaging [18]. Moreover, Xu et al. reported 3 vascular patterns: mature, hypermature, and immature [19]. They concluded that a capillary fringe and immature appearance of the membrane were associated with CNVM doubling, while lesions with a dead tree appearance were not associated with CNVM growth [19]. Karacorlu and colleagues classified active lesions as having a well-defined or ill-defined membrane appearance and reported that long-filamentous membranes were associated with inactive lesions [7]. In the light of these findings, the dead tree membrane morphology and long-filamentous networks were proven to be associated with chronic inactive lesions $[7,8,18,19]$. In the current study, consistent with the literature, $71.4 \%$ of the non-exudative eyes had a dead tree membrane morphology.

El Ameen et al. described a dark area around type 2 CNVMs in the choriocapillaris layer, based on OCTA, which corresponds to the dark ring surrounding the CNVM in the early frames of FFA and indocyanine green angiography, presenting a halo-like shape. They reported this perivascular dark halo in all type 2 CNVM patients [14]. Miere et al. reported 2 types of hyporeflective features, including a large flow void and perivascular dark halo around the CNVM in patients with subretinal fibrosis secondary to AMD. In the 49 eyes they evaluated, $63 \%$ had a large flow void and $65 \%$ had a perivascular dark halo, but they concluded that these patterns did not differ between eyes with exudative or non-exudative CNVMs [8]. In our study, exudative and non-exudative CNVMs were analyzed qualitatively, and among the exudative CNVMs, 22 eyes (70.9\%) had perivascular halo and 22 eyes (70.9\%) had loop anastomoses. This was significantly different from the incidence in non-exudative CNVMs. 
The strengths of the study were the flow area calculations of the CNVMs that were performed automatically by the device and that all of the study participants were followed by the same retina specialist at a single tertiary referral center. However, there were some limitations to the study. First, patient cooperation is crucial for achieving good OCTA images. Images with a low signal strength index can affect quantitative measurements. While measuring the size of the CNVMs, the margins of the lesion were selected manually by the observer because the software of the device could not calculate the size automatically, and all the study participants were evaluated by only 1 observer who was not blinded to the study group. Another limitation of the study was that indocyanine green angiography imaging was not performed; only SD-OCT, FFA, and OCTA were used. Patients with polypoidal choroidal vasculopathy were diagnosed only based on OCTA and SD-OCT features [20]. The small sample size is another limitation of the study. Furthermore, we did not evaluate vessel density, fractal dimension, or lacunarity, other than the size and the flow area of the CNVMs. In future, stronger prospective studies should be conducted by increasing the sample size and evaluating the effect of anti-VEGF treatment on CNV morphology and size.

\section{CONCLUSIONS}

OCTA allows clinicians to evaluate CNVMs qualitatively and quantitatively. It also provides objective documentation about the behaviors of these membranes. A perivascular dark halo around CNVMs observed by OCTA could be an activity criterion for exudative membranes.

\section{ETHICAL DECLARATIONS}

Ethical approval: All procedures performed in studies involving human participants were in accordance with the ethical standards of the institutional and/or national research committee and with the tenets of the 1964 Declaration of Helsinki and its later amendments or comparable ethical standards. The study protocol was approved at the department level, and informed consent was obtained from all individual participants included in the study.

\section{Conflict of interest: None.}

\section{FUNDING}

None.

\section{ACKNOWLED GEMENTS}

This study was presented at the European VitreoRetinal Society (EVRS) meeting, 2019 in Lisbon, Portugal.

\section{REFERENCES}

1. Witmer AN, Vrensen GF, Van Noorden CJ, Schlingemann RO. Vascular endothelial growth factors and angiogenesis in eye disease. Prog Retin Eye Res. 2003;22(1):1-29. doi: 10.1016/s1350-9462(02)00043-5 pmid: 12597922

2. Resnikoff S, Pascolini D, Etyáale D, Kocur I, Pararajasegaram R, Pokharel GP, Mariotti SP. Global data on visual impairment in the year 2002. Bull World Health Organ. 2004;82(11):844-51. Epub 2004 Dec 14. pmid: 15640920

3. Jung JJ, Chen CY, Mrejen S, Gallego-Pinazo R, Xu L, Marsiglia M, et al. The incidence of neovascular subtypes in newly diagnosed neovascular age-related macular degeneration. Am J Ophthalmol. 2014;158(4):769-79 e2. doi: 10.1016/j.ajo.2014.07.006 pmid: 25034111

4. Freund KB, Zweifel SA, Engelbert M. Do we need a new classification for choroidal neovascularization in age-related macular degeneration? Retina. 2010;30(9):1333-49. doi: 10.1097/IAE.0b013e3181e7976b pmid: 20924258

5. Yannuzzi LA, Negrao S, Iida T, Carvalho C, Rodriguez-Coleman H, Slakter J, et al. Retinal angiomatous proliferation in age-related macular degeneration. Retina. 2001;21(5):416-34. doi: 10.1097/00006982-200110000-00003 pmid: 11642370

6. Spaide RF, Klancnik JM, Cooney MJ. Retinal Vascular Layers Imaged by Fluorescein Angiography and Optical Coherence Tomography Angiography. JAMA Ophthalmol. 2015 Jan;133(1):45-50. doi: 10.1001/jamaophthalmol.2014.3616 pmid: 25317632

7. Karacorlu M, Sayman Muslubas I, Arf S, Hocaoglu M, Ersoz MG. Membrane patterns in eyes with choroidal neovascularization on optical coherence tomography angiography. Eye (Lond). 2019;33(8):1280-1289. doi: 10.1038/s41433-019-0415-1 pmid: 30932032

8. Miere A, Semoun O, Cohen SY, El Ameen A, Srour M, Jung C, et al. Optical Coherence Tomography Angiography Features of Subretinal Fibrosis in Age-Related Macular Degeneration. Retina. 2015;35(11):2275-84. doi: 10.1097/IAE.0000000000000819 pmid: 26457397

9. Farecki ML, Gutfleisch M, Faatz H, Rothaus K, Heimes B, Spital G, et al. Characteristics of type 1 and 2 CNV in exudative AMD in OCT-Angiography. Graefes Arch Clin Exp Ophthalmol. 2017;255(5):913-921. doi: 10.1007/s00417-017-3588-y pmid: 28233061

10. Muakkassa NW, Chin AT, de Carlo T, Klein KA, Baumal CR, Witkin AJ, et al. Characterizing the Effect of Anti-Vascular Endothelial Growth Factor Therapy on Treatment-Naive Choroidal Neovascularization Using Optical Coherence Tomography Angiography. Retina. 2015;35(11):2252-9. doi: 10.1097/IAE.0000000000000836 pmid: 26457400

11. Kuehlewein L, Bansal M, Lenis TL, Iafe NA, Sadda SR, Bonini Filho MA, et al. Optical Coherence Tomography Angiography of 
Type 1 Neovascularization in Age-Related Macular Degeneration. Am J Ophthalmol. 2015;160(4):739-48 e2. doi: 10.1016/j. ajo.2015.06.030 pmid: 26164826

12. Soomro T, Talks J, Medscape. The use of optical coherence tomography angiography for detecting choroidal neovascularization, compared to standard multimodal imaging. Eye (Lond). 2018;32(4):661-672. doi: 10.1038/eye.2018.2 pmid: 29600987

13. Lupidi M, Cerquaglia A, Chhablani J, Fiore T, Singh SR, Cardillo Piccolino F, et al. Optical coherence tomography angiography in age-related macular degeneration: The game changer. Eur J Ophthalmol. 2018;28(4):349-357. doi: 10.1177/1120672118766807 pmid: 29623720

14. El Ameen A, Cohen SY, Semoun O, Miere A, Srour M, Quaranta-El Maftouhi M, et al. Type 2 Neovascularization Secondary to Age-Related Macular Degeneration Imaged by Optical Coherence Tomography Angiography. Retina. 2015;35(11):2212-8. doi: 10.1097/IAE.0000000000000773 pmid: 26441269

15. Cohen SY, Creuzot-Garcher C, Darmon J, Desmettre T, Korobelnik JF, Levrat F, et al. Types of choroidal neovascularisation in newly diagnosed exudative age-related macular degeneration. Br J Ophthalmol. 2007;91(9):1173-6. doi: 10.1136/bjo.2007.115501 pmid: 17383997

16. Mentes J, Yildirim S. Optical Coherence Tomography Characteristics of Quiescent Type 1 Neovascularization in Eyes with Nonexudative Age-related Macular Degeneration. Turk J Ophthalmol. 2019;49(2):84-88. doi: 10.4274/tjo.galenos.2018.58908 pmid: 31055893

17. Zhao Z, Yang F, Gong Y, Yu S, Liu H, Wang H, et al. The Comparison of Morphologic Characteristics of Type 1 and Type 2 Choroidal Neovascularization in Eyes with Neovascular Age-Related Macular Degeneration using Optical Coherence Tomography Angiography. Ophthalmologica. 2019;242(3):178-186. doi: 10.1159/000497491 pmid: 31195396

18. Coscas GJ, Lupidi M, Coscas F, Cagini C, Souied EH. Optical Coherence Tomography Angiography Versus Traditional Multimodal Imaging in Assessing the Activity of Exudative Age-Related Macular Degeneration: A New Diagnostic Challenge. Retina. 2015;35(11):2219-28. doi: 10.1097/IAE.0000000000000766 pmid: 26398697

19. Xu D, Davila JP, Rahimi M, Rebhun CB, Alibhai AY, Waheed NK, et al. Long-term Progression of Type 1 Neovascularization in Age-related Macular Degeneration Using Optical Coherence Tomography Angiography. Am J Ophthalmol. 2018;187:10-20. doi: 10.1016/j. ajo.2017.12.005 pmid: 29269100

20. Cheung CMG, Yanagi Y, Akiba M, Tan A, Mathur R, Chan CM, et al. Improved Detection and Diagnosis of Polypoidal Choroidal Vasculopathy Using a Combination of Optical Coherence Tomography and Optical Coherence Tomography Angiography. Retina. 2019;39(9):1655-1663. doi: 10.1097/IAE.0000000000002228 pmid: 29927796 